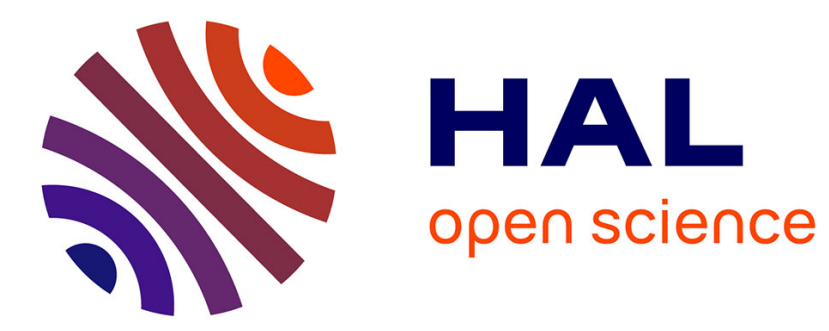

\title{
Personne et personnalisation chez Henri Wallon
}

Philippe Malrieu

\section{To cite this version:}

Philippe Malrieu. Personne et personnalisation chez Henri Wallon. Enfance, 1979, 32 (5), pp. 381-391.

halshs-01086169

\section{HAL Id: halshs-01086169 \\ https://shs.hal.science/halshs-01086169}

Submitted on 22 Nov 2014

HAL is a multi-disciplinary open access archive for the deposit and dissemination of scientific research documents, whether they are published or not. The documents may come from teaching and research institutions in France or abroad, or from public or private research centers.
L'archive ouverte pluridisciplinaire HAL, est destinée au dépôt et à la diffusion de documents scientifiques de niveau recherche, publiés ou non, émanant des établissements d'enseignement et de recherche français ou étrangers, des laboratoires publics ou privés. 


\title{
Personne et Personnalisation ${ }_{*}+1$ chez Henri Wallon
}

\author{
par
}

\section{Philippe MALRIEU}

Dans les traités de psychologie ne manque jamais le chapitre consacré à la personnalité. On y traite des activités par lesquelles les impressions venues des multiples horizons du monde extérieur et de l'organisme sont situées les unes par rapport aux autres, les réactions effectuées pour leur répondre organisées en systèmes plus ou moins délimités et articulés entre eux dans le temps de vie. On s'interroge sur les processus des synthèses psychologiques diverses qui interviennent, par exemple, pour constituer le sentiment de l'unité du corps propre, pour sérier les souvenirs en une trame définie, pour différencier le moi du non-moi comme une instance qui transcende ses états, ou pour s'affirmer soi-même comme une fonction d'ordre, de choix, de décision.

Bien que Wallon, dès sa thèse de médecine, se soit souvent préoccupé de ces problèmes, ils nous semblent distincts de ceux qui l'intéressent lorsqu'il parle, non plus de personnalité, mais de personne. Ainsi dans l'Évolution psychologique de l'enfant présente-t-il cette dernière comme un des quatre "domaines fonctionnels" de la vie psychologique, à côté de l'affectivité, de la motricité et de la connaissance. Non sans avoir remarqué que la distribution des activités entre eux "ne va pas sans doute sans quelque artifice" (p. 122): car celles d'un domaine défini dépendent de celles des autres, et en une certaine mesure les déterminent en retour, bien que chacune, de par son inscription psychologique comme en vertu de sa fonction sociale, possède sa spécificité. Quelle est donc la spécificité de la personne selon Wallon? Une première définition qu'il en donne (p. 127) renvoie à l'idée qu'elle consiste en un "sentiment de dignité ", dans "l'amalgame", aux "réflexes" d'adaptation au milieu, de "valeurs dont l'unique support consiste en notions toutes abstraites ou idéales "... Cette définition s'éclaire, au chapitre de la Personne (p. 204-213), de l'étude de sa formation, où Wallon met l'accent, fondamentalement, sur les étapes et les processus de ce qu'on peut appeler la désaliénation du sujet: différenciation des autres et de soi, désengagement des objets habituels, conquête corrélative d'un domaine de biens propres, socialement reconnus comme tels, distinction de ce qu'il doit paraitre et de ce qu'il peut être, libération de ses infériorités par les identifications, et libération de celles-ci par l'expérience de ses multiples possibilités singulières, qui le font différent des autres et qu'il défend contre les empiétements des autres: "Moi je ne suis pas... lui, toi, eux $»$. 
Nous saisissons, au travers de ce recensement succinct de quelques unes des (désalienationss, par la personne, pourquol Wallon déclare que (a personne n'arrive a se constituer qu'a travers l'ensemble des autres etapes fonctionnelles: (p. 127), et pourquoil la considere comme indispensable, inhérente, aux progrès dans les autres domaines. D'une part en effet cette série de désengagements-de lémotion, des conditionnements, des attachements aux personnes, de la conscience morcelée qu'il a de lui-même, de ses personnages... n'est possible que par l'avènement de mutations, dans l'affectivité grâce aux progrès moteurs et cognitifs, dans les connaissances par l'avènement d'une motricité et d'une affectivité transformées: mutations dont les instruments se trouvent dans la maturation et dans les rapports avec la culture. D'autre part les progrès de la personne, définis par ceux du sentiment de dignité et par la construction des valeurs, sont indispensables au développement des affects, des mouvement et des connaissances, car ce développement téside dans l'appropriation des régulations culturelles des activités, or cette appropriation ne peut s'effectuer que sur le terrain spécifique de la croissance de la personne, c'est a dire dans la dialectique des rapports entre les personnes, c'est dans la rencontre des autres, à la fois sujets et êtres de culture, que se réalisent les progrès de la vie affective, motrice, intellectuelle.

Une comparaison avec les recherches de Piaget permettrait de situer la perspective wallonienne. Dans ses travaux sur la perception, les images, la mémoire, le langage et aussi les conduites sociales et morales, Piaget démontre comme nt les processus intellectuels transforment ces activités, en même temps qu'ils se développent eux-mêmes dans les restructurations quils leur imposent: On pourrait dire, dans une première approximation, que si Wallon ne méconnatt pas ce que les progrès psychologiques doivent aux processus cognitifs, $c^{3} e s t$ à une unité plus fondamentale qu'il fait appel pour en rendre compte. Ce sont les différenciations d'une totalité primitive dans ses rapports à d'autres ensembles, de la personne sous ses formes premières dans ses relations à ses multiples milieux, qui commandent l'évolution psychologique. Globalement on pourrait dire: si Wallon confere à la personne le rôle que Plaget accorde aux processus intellectuels, c'est parce qu'il considère indispensable d'inscrire ces derniers dans l'ensemble des relations motrices, affectives, culturelles, où ils interviennent avec leurs progrès spécifiques (1)

Il nous faut rechercher ce qui dans les conceptions épistémologiques de Wallon justifie ce recours à la notion de personne, pour examiner ensuite sur quelques exemples comment elle intervient dans l'interprétation du développement psychologique, et de ce fait acquiert le statut d'un concept méthodologique.

(1) D'où les critiques que Wallon adresse à Piaget « il donne a l'évolution psychique une base parcellaire et trop étroite " en admettant qu'elle résulte de la combinaison « d'éléments d'abord distincts" (les schémas moteurs)-il ignore les ensembles sociaux dans lesquels se forment symbole et connaissance, et du même coup méconnaît leur spécifité, qui est de procéder de é par la représentation (De l'Acte à la Pensée , ch, 1). 


\section{LES DIALECTIQUES DE LA VIE PSYCHOLOGIQUE \\ ET LA PERSONNE}

Un des caracteres fonda mentaux de la méthode de Wallon réside dans son part pris de prendre en compte et de comprendre ce qu'll y a d'original, de nouveau dans chacune des conduites humaines. En quol il reconnait la part de verité qu'il y a cans les théories spiritualistes qui se refusent aux explications réductrices du «supérieur s par «'inférieur »-de limage par une $\mathrm{trace}$ s perceptive, de la pensée conceptuelle parlassociation d'images, de la décision par le jeu des tendances, en même temps qu'il se refuse a voir dans le « supérieur s un fait premier, qui pourrait être soustrait aux investigations déterministes.

Reconnaitre 1 originalité d'un comportement par rapport a ceux qui le précedent dans lontogénèse requiert, au plan de la méthode, que, procédant par opposition, on fasse apparaître sous les ressemblances manifestes lexistence de différences profondes Ainsi par exemple procède Wallon a propos des emotions et des automatismes, de lintelligence pratique et de 1 intelligence discursive, du symbole et du signe. Il ne nie certes pas qu'au cours de l'évolution interviennent des processus constants tqua chaque étape on puisse repérer l'action du conditionnement, de la maturation, de lalternance entre assimilation et accommodation, des conflits entre pulsions et interdits sociaux. Mais ces processus, invoqués tour à tour par les diverses écoles pour rendre compte du psychisme, passent a côté des caractères spécifiques de chaque type d'activité qu'on voit apparaître au cours de l'évolution. Ce qui importe à Wallon, c'est de saisir comment se produit l'innovation psychologique.

La réponse, au-delà de quelques textes où il accorde un rôle au hasard, a la rencontre de séries causales indépendantes, il la trouve dans la perspective dialectique du dépassement des conflits, telle qu'elle ressort dès 1920-30 de sa réflexion sur le statut de la psychologie, de sa définition du psychisme « L'homme psychique se réalise entre l'inconscient biologique et l'inconscient social. Il les intègre diversement entre eux. Mais s'il veut se connaître il doit établir ses corrélations avec l'un et avec l'autre s (2), on y perçoit une quadruple dialectique:

1 Dialectique dinteraction entre les fonctions biologiques et les régulations sociales, celles-ci obéissant à la demande des premières, non sans réagir sur elles l lorganisation sociale vise à satisfaire les besoins, mais les réoriente, en produit de nouveaux, et parfois en vient à les opposer les uns aux autres.

2 Dialectique des réactions et du milieu dans ses activités pour satisfaire ses besoins innés ou acquis, lêtre vivant transforme le milieu dont il dépend, introduisant ainsi des déséquilibres nouveaux, quil lobligent à transformer ses propres réactions.

3 . Or, chez lhomme cette double restructuration passe souvent par des prises de conscience de niveaux divers on y voit affleurer la dialectique de linconscient et du conscient. Chaque fois en effet que s'installe un déséquilibre (ainsi chez l'adolescent qui ne peut satisfaire ses besoins-désirs dans

(2) Science de la nature et science de l'homme la psychologie, in Revue de Synthese, octobre 1931 (texte daté du 28 maí 1930, repris dans Enfance, 1959, no 3-4, p. 219) 
les cadres sociaux où, enfant, il inscrivait ses comportements), des aspects nouveaux se decouvrent aussi bien dans le milieu que dans l'individu, aspects jusqualors méconnus, qui exigent la constitution de réactions nouvelles, mais celles-ci ne peuvent s'installer sans une lutte contre les réactions anciennes : elles «doivent se constituer aux dépens des premières en les réorganisant différemment" (3). Ces conflits sont le lieu de prises de conscience émotionnelles - puisqu'ils provoquent le désarroi des réactions constituées, l'étonnement, l'angoisse ou l'enthousiasme - et de prises de conscience cognitives - en permettant la révélation d'aspects ignorés, d'où résulte la restructuration des connaissances constituées. $D^{\prime}$ 'où résulte aussi un remaniement des activités inconscientes tant biologiques que sociales. Biologiques quand les réactions instinctives ou habituelles sont remodelées dans les cadres sociaux, techniques ou linguistiques par exemple;-sociales, quand les règles des institutions sont modifiées au profit d'un développement plus complet des potentialités organiques.

4. Où se manifeste un autre aspect de la dialectique psychologique: celle de la réaction et de l'acteur: "Toute réaction peut marquer comme une extension de l'être psychique. Il dépend d'elle comme elle dépend de lui » (4). Dans la mesure en effet où il opère un progrès dans l'intégration du conscient et de l'inconscient, du social et du biologique, il s'assure une extension de lui-même, en surmontant les clivages qui existent entre les impulsions et les instruments organiques d'une part, les consignes, les contraintes, les idéaux sociaux d'autre part.

Dans ces prises de position en faveur d'une conception dialectique du psychisme, nous voyons tout à la fois la marque d'une série de prises de position épistémologiques et la reconnaissance de l'intervention d'une fonction de personne qui a son originalité par rapport à toutes les autres.

Wallon s'oppose à tous les mécanismes. Il refuse l'hypothèse qu'« en dernier ressort " on pourrait rendre compte des activités humaines par des processus psychologiques, ou par des processus sociaux. Il refuse de considérer le psychisme comme une composition de réactions élémentaires, par des processus de conditionnement, par des associations, de plus en plus complexes.

Wallon par ailleurs s'oppose aux psychologies, florissantes au début du siècle, qui en se fondant sur l'introspection concluent à l'initiative de La Conscience et du Moi, conçues comme des puissances ordonnatrices de la représentation et de l'action. Comme il se méfie des théories dynamistes, telles les psychanalyses, qui placent dans des forces concourantes ou opposées le principe du développement psychologique: dans ces deux courants il trouve la présence d'une conception substantialiste incompatible avec la méthode scientifique.

Les mécanismes comme les substantialismes méconnaissent l'évolution psychologique, l'avènement de formes radicalement nouvelles d'activités, et l'origine de cet avènement dans les conflits qui traversent le réseau de nos activités - conflits entre l'organisme et ses milieux, entre le biologique et le social, entre les processus inconscients et conscients.

(3) Les origines du caractère (éd. 1949. P.U.F), p. 58-59.

(4) Sc. n/Sc. h, ibid., p. 217 
Cest précisément en ce leu de conflits qu'il lui semble nécessaire de déceler la formation et l'intervention de la personne, en tant que fonction de position et de résolution de ces conflits. Quelques définitions de lui d'abord pour orienter nos remarques.

Dans la vie mentale (8.54.3), il affirme au chapitre Formation de la Personne que "la conscience personnelle du sujet est une acquisition relativement tardive", et ajoute: "Le point de vue de la personne est lié à une affirmation d'identité qui l'oppose comme telle à chacune de ses expériences particulières et successives, qui oppose le sujet au contenu de son activité ou de ses représentations et aux événements de sa propre existence ». Cette affirmation d'identité est conquise dans une lutte à double face: contre la fusion aux situations et aux objets, au travers de crises où le sujet en s'opposant aux autres conquiert son autonomie; - et contre la dispersion de ses actes, dans un processus "d'intégration des facteurs biopsychiques qui l'animent aux objectifs de sa vie ": aux valeurs, dont Wallon, nous l'avons vu, fait l'instrument de la découverte de notre " raison d'être", de la conscience que nous avons un "destin" (ibid 8.54.4).

Opposition et intégration, ces deux processus sont présents à tous les âges. A 3 ans, mais aussi entre 7 et 12 ans, lorsque la personne parvient à la phase catégorielle, où le sujet en passant d'un rôle à un autre, en classe, dans la famille, avec les camarades, doit lutter contre la fixation à l'une de ses positions pour "se connaître comme simultanément le lieu de diverses possibilités" (Évolution psychologiques p. 211). A l'adolescence également, lorsque, en s'opposant aux autres, "aux relations diverses de société où la personne semblait s'être effacée, elle cherche une signification", et la trouve dans les valeurs qui lui permettent de justifier ses choix (ibid. p. 212-3).

D'où nous pourrions sans doute tirer une définition provisoire: « $\mathrm{La}$ personne consiste dans les activités par lesquelles le sujet, en relation d'assujetissement et d'opposition aux autres, s'installe dans les conflits où l'entrainent les incitations des inconscients biologique et social, et s'attache à les surmonter, en se mettant à distance des événements vécus, en se découvrant comme un lieu de possibles, en subodonnant ses actes à des valeurs".

\section{FORMATION ET ACTION DE LA PERSONNE}

Comment cependant parvenir à l'utilisation scientifique de cette notion de personne, s'il est vrai qu'on ne peut comprendre intégralement les. conduites humaines sans considérer le travail de personne comme une de leurs variables indépendantes (interdépendantes, vaudrait-il mieux dire)?

On prendra chez Wallon trois exemples d'analyse pour définir comment peut se concevoir la genèse réciproque des conduites et de la personne:

- aux sources du symbolique,

- dans l'installation d'une temporalité de vie,

- aux origines de la pensée.

\section{L'avènement du symbolique.}

S'agit-il de comprendre comment l'enfant vers 2 ans parvient, pour "représenter"sa mère, à se faire représenter lui-même par une poupée? On 
découvre a longine du jeu de fiction une série de conflits, qui remontent aux premiers mois de la vie. Ce qulil manifeste clairement, c'est une fonction de dédoublement (du moi en la mère, du noi en poupee). Les origines peuvent être cherchées dans les jeux dalternance et de réciprocite qui ont commence vers 4 a 5 mois, ou tour a tour l'adulte et lentant jouent un róle d'émetteur et de récepteur (échangeant un cri, un geste, plus tard un objet). Lenfant y apprend à devenir un centre d action sur autrut une des premières activités de personne, qui s'affirme quand il se sert de son geste pour susciter attention et réponse d'autrui (ainsi il jette un objet dans l'intention qu'il soit ramassé), il agit sur luim même pour agir sur autrui.

Cette premiere libération a l'égard des réactions a fondement de conditionnement, des réactions circulaires, des réponses émotionnelles lengage dans des conflits où se forme une première forme de personne. conflits internes entre les conduites anciennes ef les conduites naissantes, conflits avec les autres.

Ces derniers ont été les plus remarqués, notamment par les psychanalystes, qui en ont donné une interprétation restrictive (stade sadico-anal). Les oppositions aux autres sont des réactions à ses propres échecs au cours des tentatives qu'il fait pour les faire agir selon ses désirs, pour devenir autonome. Ses agressions sont le témoignage d'un sentiment d'incertitude sur ses pouvoirs, sur l'acceptation ou le refus, par autrui, de ses initiatives aventureuses, incompatibles avec les habitudes des adultes.

Mais le désir d'autonotmie a des sources plus profondes que la résistance aux contraintes interpersonnelles. II est relatif aux grandes conquêtes que l'enfant réalise en cette période de l'instrument, du corps propre, du mol, du langage, de la représentation. Conquêtes interdépendantes, non sans que des conflits se manifestent entre elles terrain de la formation des premières conduites de personne, ou s'approfondit le dédoublement inauguré dans les jeux de réciprocité comme « prend-donne, , coucou -ah le voilà !s.

Les instruments, outils des plus simples techniques. le bâton, le "panier», la balle, incitent a finaliser l'action, à contrôler l'ajustement du mouvement - moyen au résultat, à distribuer le geste en moments. Ils élaborent les attitudes de l'intelligence des situations : " pouvoir d'assembler, parmi les impressions du moment, toutes celles qui peuvent contribuer a la réussite de l'action en lui fournissant les moyens adéquats " (De l'Acte à la pensée p. 16). Bien qu'il n'y ait en elle «qu'attraction mutuelle du réel et des impulsions ", elle réalise une extension temporelle des comportements sur les choses qui est un aspect de l'extension de l'être psychique.

Elle est un des lieux de la conquête du corps propre, qui requiert l'élaboration de cet espace encore subjectif instauré dans l'intelligence des situations celle-ci en effet inaugure la situation du corps dans ses relations aux objets. Mais c'est surtout dans les imitations que l'enfant apprend a se donner de son corps un systeme de percepts et d'images en projetant sur ses mouvements la perception qu'il a du corps d'autrui, il parvient a une certaine objectivation du sien, qui se précise dans la comparaison des images spéculaires de soi et d'autrui (mais qui n'en découle pas).

Simultanément l'enfant, au cours de la deuxième année, entre dans le langage et dans ses problèmes. Il y entre, dans la compréhension dautrui, 
dans la mesure ou ll desire participer aux actions de celul cl, se mettre a sa place notivation premiere des lietux qu'll établit entre les signifiants entendus et les référents. De mème qu'll parle, non seulement pour manifester des désirs, mais pour se poser en interlocuteur, capable d'utliser le langage et les atútudes qu'il véhicule désignation, refus, désir, accord, différenciation, assimilation, énonciation., dans une affirmation de soi devant autrui.

Conquete des instruments, du corps propre, du langage, condition et conséquence des processus de personnalisation quil se développent de $1 \mathrm{a}$ 3 ans, au cours des relations interpersonnelles que Wallon analyse dans les deux dernières parties des Origines du Caractère. Il y montre le jeu conflictuel d'une participation de lenfant-sympathie, identification-aux actions, aux affects, aux positions des autres, et dune opposition agression, falousie, plus tard singularisation, distinction, séparation - à leurs entreprises sur l'enfant. Dans la participation, dont découle la frénésie d'imitations de la deuxieme année, lenfant développe son champ d'actions en même temps que le contrôle, venu des modèles externes, de ses conduites. Mais ce contrốle même, qui s'exerce dans l'utilisation des instruments, la maitrise du corps propre, les différenciations linguistiques, l'invite à se séparer des autres, à se dégager de l'emprise qu'ils exercent sur lui, sur un mode émotionnel é sur un mode représentatif c'est ce qu'il fait dans ses. oppositions et dans la définition, encouragée par les adultes, d'un proprium. son nom, ses objets, ses habitudes, ses souvenirs, ses rồles dans les groupes auxquels ils participe, bientôt ses projets de dépassement.

Dans cette dialectique de la sympathie et de la séparation, l'enfant « en se retirant de ce qui n'est pas (lui-mểme), en dissociant l'expérience qu'(il) vit suivant la catégorie du moi et du non-moi», effectue « un dédoublement vis à vis de l'intuition concrète " (1). Dédoublement dont le symbole est à la fois l'expression et l'instrument. Ainsi la poupée, en le u représentant s, est-elle le moyen pour l'enfant de représenter la mère, et en s'identifiant a elle d'atteindre à une certaine indépendance vis à vis d'elle, puisqu'il la remplace par lui-même le symbole répond exactement a l'exigence de ce dédoublement dans laquelle je $n$ 'existe qu'en se distanciant de la personne à laquelle il s'identifie.

Cet acte de représentation symbolique est bien un " travail de personne ": il prend appui sur les premiers dialogues préverbaux de la première année, et se nourrit de tous les échanges qui lui font suite. Il est présent dans les restructurations des activités instrumentales, ou se développe un espace de possibles qui va servir de cadre aux conduites de détour. ll est présent dans la construction de la représentation du corps propre, dont le corps dautrui, les images dans la glace, les portraits constituent autant de symboles épars. Ce travail est présent aussi dans le langage le mot, avant d'être un signe inséré dans un réseau d'opposition, a dans ses premiers emplois un statut de symbole conventionnel, que l'enfant adopte pour signifier son statut d'alter-ego. Si bien qu'on peut penser à un processus d'interstructuration. l'attitude symbolique, sous-tendue par les relations interpersonnelles et les dédoublements qui les constituent, pénètre les grandes conquêtes culturelles que réalise l'enfant Mais il faut voir, également que celles-ci

(1) Origines du Caractère, p. 219 
-instrument, corps propre, langage - ont été élaborées au cours de l'histoire sur le fondement de l'aptitude symbolique: elles constituent pour l'enfant une incitation permanente à développer l'attitude symbolique.

\section{Temporalisation et personne.}

Mémoire de soi, conscience des progrès accomplis dans l'autonomie, projets, constituent des étapes de la conscience de soi, indispensables à l'avènement de la conscience du temps de vie, et d'une sorte d'identité de soi dans le temps. Ces étapes sont relatives, à plusieurs égards, aux relations de l'enfant aux personnes et à la situation de soi par rapport aux autres.

Il faut pour le saisir revenir à l'idée fondamentale que «le Moi et l'Autre se constituent conjointement" (5) dans la dialectique d'identification et de séparation dont nous venons de parler. De 3 à 6 ans déjà, l'identification à des adultes ou à des êtres mythiques prestigieux assure à l'enfant une voie pour surmonter les blocages auxquels le condamne la conscience de ses échecs: alors apparait vers 4 ans "le souci de présenter (le Moi) à son avantage, de le faire valoir et de s'admirer soi-même ", suivi vers 5 ans d'un «besoin d'amplification qui fait prendre autrui pour modèle à supplanter i. (ibid. p. 89-90). Les relations interpersonnelles l'engagent donc dans une perspective d'avenir, perçu de façon égocentrique, qui a pour corrélat (avec l'aide de son entourage) la construction d'une mémoire des événements, heureux ou malheureux, fixés et rappelés comme témoignage des difficultés surmontées, des infériorités dépassées.

Ce travail d'inscription de soi dans le temps se complique lorsque vers 8-9 ans, "sa personne (entre) dans la phase catégorielle", lorsqu'il se connaît lui-même comme simultanément le lieu de diverses possibilités " (6), dans les différents groupes dont il fait partie, et dans ceux où il sait devoir s'intégrer plus tard. Il en vient alors, vers 10-12 ans, à formuler des jugements de valeur sur les conditions d'existence des adultes, pour refuser certaines d'entre elles, en ambitionner d'autres, et pour s'ouvrir à lui-même un champ. de perspectives larges, dans le cadre de la vie quotidienne ou du métier, où rien n'est encore défini, où tout peut se réaliser. Ce n'est qu'après la puberté, de façon différente selon les filières scolaires et culturelles, que les urgences successives, les choix inévitables vont obliger l'adolescent à "s'attribuer un destin ", à inscrire ses conduites dans un temps de vie personnel.

Ces transformations dans la situation temporelle de soi ne se conçoivent pas indépendamment d'une représentation du temps qui elle-même est tributaire des progrès cognitifs, Wallon l'a montré dans Les Origines de la. pensée chez l'enfant (7). Elles dépendent aussi largement des informations fournies par les adultes sur le passé et l'avenir social de l'enfant. Mais si on admet avec Wallon que "le temps a pour composantes indissolublement complémentaires le fait subjectif et l'ordre objectif de la succession ", il faut alors reconnaître que le temps vécu, depuis son origine dans les premières "impatiences" (8) jusqu'aux aspirations de l'idéal du moi, est tissé dans l'expérience de ses déplacements sur les positions multiples des autres, qui sont le lieu privilégié de la personnalisation.

(5) Niveaux et fluctuation du Moi. Evol. Psychiatrique, 1956 (in Enfance 1963, n 1-2, p. 97).

(6) Evolution psych. de l'enfant, p. 211.

(7) Edit, de 1945. t. II, p. 206 sq., p. 302-309.

(8) Origines de la pensée, ibid. p. 303. 
La personne aux origines de la pensée.

«C'est le signe qui fait acceder au plan de la représentation vraie ». Le signe différencie les référents en fonction de ce que la pratique collective en sait. Chaque phrase est prise de position sur ce qui est, négation de ce qui n'est pas. Où on peut reconnaître une manifestation de la personne: saffirmant devant les autres, capable de distinguer le vrai du faux, et véridique. Le monde des signes introduit à l'édification des «disciplines mentales » en défaut chez l'enfant «turbulent": inhibition, effort contre l'échec, obéissance à la consigne... (9).

Pourtant, le langage ne fonde pas la pensée. Celle-ci est la mise en relation de réalités. En sa première manifestation, dans le "couple ", on y voit jouer, de 4 à 8 ans, les exigences et les insuffisances de la pensée de l'enfant:

«La pluie, c'est du vent... Le vent c'est quand il fait froid ». Le couple témoigne d'une quête des «raisons" du phénomène. D'où vient ce désir de lier? - Il faut remarquer qu'il intervient, d'abord, de la relation interpersonnelle où il se produit. Ce peut être, comme dans les Origines de la Pensée, une question de l'adulte à l'enfant. Elle le contraint à sortir du phénoménisme: la pluie, il la voit, l'accepte ; quand on lui demande ce qu'elle est, le voilà déporté hors du vécu, dans une sphère de pensée qui contient la notion vague d'une genèse, vers la recherche des antécédents et des conséquents. La question l'oblige à se mettre à distance de son vécu, parce qu'il doit intérioriser le point de vue d'autrui: première manifestation d'un travail de personne dans la construction des connaissances.

Mais le dialogue, comme dans les Origines de la Pensée, a un deuxième résultat: il met l'enfant en présence de ses contradictions. S'il a défini le vivant par l'acte' de manger, et s'il reconnaît que la plante mange la terre, pourquoi lui refus-t-il d'être vivante? Le dialogue révèle qu'il y a d'autres possibilités que celle à laquelle il avait pensé : éveil du doute, et en face de l'adhésion spontanée la représentation des possibles, la réflexion.

De quoi s'agit-il avec celle-ci? - De surmonter le syncrétisme. L'enfant juxtapose, pour rendre compte des phénomènes, des jugements issus de ses expériences parallèles. De ce qu'il a perçu (le vent c'est le froid; ou: c'est les arbres). De ses rêveries autour d'un mythe (le vent c'est la respiration du bon Dieu). De savoirs pénétrés d'imaginaire: (ce sont les vagues de l'océan qui font le vent). Le syncrétisme - incapacité à objectiver et confronter les sources de ses représentations - est le fait d'une personnalité morcelée, incapable de coordonner ses activités et ses jugements. La critique qui va. le réduire constitue le travail intellectuel de la persónne. Travail en coaction avec l'adulte, qui initie l'enfant à l'utilisation des instruments cognitifs élaborés au cours de l'histoire. Tels que : l'inventaire, la comparaison, la définition, la situation des objets et des événements dans l'espace et dans le temps, la causalité, l'action réciproque.

Mais l'enfant ne parvient à maîtriser ces instruments qu'avec difficulté. Wallon relève les divers types de confusion qu'il commet. Il se contredit, faute de confronter ses jugements: les vagues font le vent dans un certain cadre, le vent fait les vagues dans un autre. L'activité par laquelle la contra-

(9) Evol. psycho, de l'enfant, p. 78-102. 
diction se révèle a lui est un acte de personne, ll apprend de lexpérience du mensonge ce qu'est la contradiction, et ce qu'est la vérité. Quand il juxtapose des vérités contradictoires, son embarras révele qu'il commence a prendre conscience que la logique est une obligation, que a la connaissance ne serait pas connaissance st elle n'était pas normatives.

1 y a interstructuration entre 1 activité critique qui dégage 1 enfant de ses interprétations subjectives et son attachement de personne aux valeurs de vérité qui lui permettent de surmonter les divisions de sa personnalité Ainsi se manifeste, dans ses échanges avec les initiateurs au savoir vral, la causalité des valeurs rationnelles, telles qu'elles ont été constituées au cours de la recherche philosophique et scientifique, La personnalisation, c'est de ce point de vue le travail effectué dans le sujet parle corps des vérites constitués, et sa mise a l'épreuve dans des expériences concretes.

On peut saisir, dans la succession de ses travaux, comment Wallon parvient à faire de la personne une fonction comparable à l'affectivité, a la motricité, a la connaissance.

Dans l Enfant turbulent, il s'attachait, a l'encontre des psychologies de l'introspection et du postulat d'une force psychique organisatrice du donné, a montrer l'ensemble évolutif que constitue lorganisme, les actions combinées, hiérarchisées des sous-systèmes qui le composent. Mais il apparaissait alors que ce réseau organique ne fonctionne qu'entraîné par le sujet dans ses relations a ses multiples milieux, sur le fondement des postures, germes des régulations des personnes. Or, les Origines du Caractère le soulignent dans l'Avant-Propos, ces actions chez l'homme sont de société, sont des coactions avec des individus de culture. Ce qui amenait Wallon à reconnaître que non seulement les conduites humaines sont instituées et nommées, comme le disaient Durkheim ou Levy -Bruhl, mais que le corps lui-même est organisé par la société il est « des champs corticaux, tel celui du langage, qui impliquent la société comme les poumons d'une espèce aérienne impliquent l'existence de l'atmosphère i.

Mais il n'y a pas lieu de faire de ce réseau social une cause premiere. Les normes sociales sont impliquées dans des contradictions, vécues au niveau des relations interpersonnelles, où elles restructurent. Il faut admettre un troisième ensemble:

Entre ces deux systemes de conditions (organiques et sociales), une marge est ouverte à lactivité de tous et de chacun, où les combinaisons de buts et d'aptitudes, de réactions réciproques, d'incidences en cascades, sont susceptibles de tous les degrés entre le conformisme et le polymorphisme, entre la contrainte et la liberté $(10)$.

Systeme, non de la Personne, mais des personnes en relation, il est le médiateur entre les deux autres. Il organise le corps selon les demandes sociales, et celles-ci en fonction des exigences organiques, telles que les structure lhistoire sociale. Mais il ne joue pas seulement cette fonction d'intermédiaire: il est lui-même doté d'une sorte d'autonomie, d'une

(10) Origines de la Pensée, t. II, p. 424 
finalité. Car, nous lavons vu, les modalités de son intervention résident dans les prises de conscience, dans les réactions affectives sources des valeurs, dans les investigations cognitives qui aboukissent aux concepts, dans la création critique des ouvres. Toutes ces activités ont pour caractéristique de répondre a des conflits, et elles n'y parviennent qu'en proposant et préparant une restructuration de la société, et pour celle-ci, a longue échéance, de lorganisme lui-même. Ainsi ces activités deviennent-elles elles-mêmes des valeurs, des fins qui régissent des conduites spécifiques, comme l'éducation intellectuelle, artistique, politique...

- Le pouvoir déterminant est dans ce qui se réalise, non dans ce qui sert a le réaliser,. Dans cette remarque, opposée aux mécanismes, se trouve sans doute laxe de la théorie de la personne chez Wallon, Les systèmes biologique et social sont indispensables à la réalisation de la personne. Et pourtant elle leur est relativement extérieure, comme l'est selon Wallon, la fonction a ses organes (l'ensemble à ses parties). Elle est entre eux, la découverte des conflits qui existent entre eux, par les émotions, la question, l'imaginaire, la critique. Elle est l'instrument dialectique de leur devenir, de leur relativisation par la connaissance de leur réciprocité et de leur mise en contradictín, On peut donc bien affirmer qu'elle est "le pouvoir déterminant", dans des conditions définies. «Ce sont les hommes qui font leur histoire" - ce sont, pourrait-on dire, les personnes, dont les contradictions internes, en relation de réciprocité avec les contradictions dans et entre les ensembles organique et social, suscitent des inventions, régressives ou progressives, dont en effet l'histoire est constituée.

Universite de Toulouse-Le Mirail 